\title{
Electromagnetic mapping of the effects of deep brain stimulation and dopaminergic medication on movement-related cortical activity and corticomuscular coherence in Parkinson's disease
}

\author{
Kousik Sarathy Sridharan ${ }^{a, b, c, \#, ~ A n d r e a s ~ H ø j l u n d ~}{ }^{a, b}$, Erik Lisbjerg Johnsenª, \\ Niels Aagaard Sunde ${ }^{d}$, Sándor Beniczky ${ }^{\mathrm{e}, \mathrm{f}}$, Karen $\emptyset_{\text {stergaard }}^{\mathrm{a}, \mathrm{b}}$
}

\section{AFFILIATIONS}

aDEPARTMENT OF NEUROLOGY, AARHUS UNIVERSITY HOSPITAL, NØRREBROGADE 44, 8000 AARHUS, DENMARK

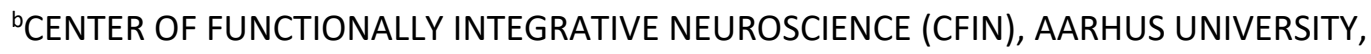
NØRREBROGADE 44, 8000 AARHUS, DENMARK

C DEPARTMENT OF BIOMEDICAL ENGINEERING, INDIAN INSTITUTE OF TECHNOLOGY, HYDERABAD 502285, INDIA

dDEPARTMENT OF NEUROSURGERY, AARHUS UNIVERSITY HOSPITAL, NØRREBROGADE 44, 8000 AARHUS, DENMARK

'DEPARTMENT OF CLINICAL NEUROPHYSIOLOGY, AARHUS UNIVERSITY HOSPITAL, NØRREBROGADE 44, 8000 AARHUS, DENMARK

'DEPARTMENT OF CLINICAL NEUROPHYSIOLOGY, DANISH EPILEPSY CENTER, KOLONIVEJ 1, 4293 DIANALUND, DENMARK

\#CORRESPONDING AUTHOR: kssi@clin.au.dk

30 PAGES, 5 FIGURES, 3 TABLES, 3 SUPPLEMENTARY FIGURES, 1 SUPPLEMENTARY TABLE TABLES AND FIGURE CAPTIONS APPENDED BEFORE REFERENCES SECTION

\section{TOTAL WORDS: 5024}

Abstract: 249 words

Introduction: Words: 653; Methods: 1915; Results: 1468; Discussion: 988 


\section{Abstract}

\section{Background}

35 Parkinson's disease (PD) is a debilitating neurodegenerative disorder. PD can be treated with deep brain

36 stimulation (DBS) when dopaminergic medication is no longer a viable option. Both treatments are

37 effective in improving motor symptoms, however, their underlying mechanisms are not fully elucidated

38 yet.

\section{Objectives}

To study the effects of DBS and dopaminergic medication treatments on cortical processing and

41 corticomuscular drive during movements.

\section{Methods}

Magnetoencephalography (MEG) was recorded in 10 PD patients and 10 healthy controls, performing

44 phasic hand contractions (hand gripping). Measurements were performed in DBS-treated, untreated and

45 dopaminergic-medicated states; healthy controls received no treatment interventions. Participants performed phasic contractions with their right hand, recorded with electromyography (EMG). Our

47 measures of interest were beta $(13-30 \mathrm{~Hz})$ corticomuscular coherence $(\mathrm{CMC})$ and low-gamma $(31-45 \mathrm{~Hz})$

48 power. We used Bayesian statistics on summary values from sensor space data, and we localized the 49 sources of the effects of treatments on beta-CMC and low-gamma power using beamforming.

\section{Results}

51 In PD patients, DBS led to reduced CMC values, whereas dopaminergic medication increased beta-CMC

52 values (localized to contralateral M1) to even higher levels than the controls. DBS, on the other hand,

53 increased low-gamma power (localized to M1) compared to controls and to other conditions. Yet both

54 treatments had similar beneficial effects on the patients' motor symptoms evaluated by UPDRS-III. 


\section{Conclusion}

56 Despite comparable improvements from both treatments on motor symptoms, DBS and dopaminergic

57 medication seem to have different effects on motor cortical function. This indicates that the treatments

58 undertake different functional strategies to improve PD symptoms.

\section{Keywords}

60 Parkinson's disease, magnetoencephalography, DBS, dopaminergic medication, phasic contractions,

61 corticomuscular coherence, gamma oscillations

\section{Introduction}

Parkinson's disease (PD) is a debilitating neurodegenerative disorder due to the dopaminergic denervation in the striatum and loss of dopaminergic neurons in the substantia nigra pars compacta, in which motor symptoms such as tremor, rigidity, akinesia and gait disturbances manifest as significant

67 disease characteristics (Hughes et al., 1992; Lang and Lozano, 1998; Hammond et al., 2007). Dopamine precursor levodopa remains the primary treatment option. But in advanced PD, motor complications arise due the so-called OFF-states where patients experience motor symptoms at sporadic time periods despite being medicated. In such cases, deep brain stimulation (DBS) of the subthalamic nucleus (STN) is an

71 alternative therapeutic recourse that generally ameliorates the motor complications (Limousin et al.,

72 1998; Østergaard and Aa Sunde, 2006; Johnsen et al., 2009) and is considered more effective in managing

73 symptoms than dopaminergic medication alone (Deuschl et al., 2006).

74 Both DBS and dopaminergic medication alleviate the motor symptoms, but the underlying mechanisms

75 of DBS, in particular, are still unclear. Several possible mechanisms of DBS have been proposed, and the 76 evidence points to a modulation of the motor cortical network activity as the main driver of symptom 
77

78

79

80

81

82

83

84

85

86

87

88

89

90

91

92

improvements (Mclntyre et al., 2004; Schnitzler and Gross, 2005; Mclntyre and Hahn, 2010). Such a modulation likely happens through antidromic activation of the so-called hyperdirect pathway between M1 and STN (Nambu et al., 2002; Gradinaru et al., 2009; Whitmer et al., 2012).

Outside the basal ganglia network, motor cortex is thus key in understanding the potential differences in effect mechanisms of DBS and dopaminergic medication. In this context, assessing treatment effects on movement related oscillations in the cortical mantle and on corticomuscular communication allow for probing exactly such potential differences.

Corticomuscular coherence $(\mathrm{CMC})$ is known to have influences of both descending and ascending motor pathways (Riddle and Baker, 2005; Baker et al., 2006; Witham et al., 2011) and plays a crucial role in corticomuscular communication (Baker et al., 1997; Salenius et al., 1997; Kilner et al., 2000; van Wijk et al., 2012). Beta-CMC is reduced in untreated PD patients (Salenius et al., 2002). DBS seems to affect betaCMC variedly with a high degree of inter-subject variability (Park et al., 2009; Airaksinen et al., 2015b) whereas dopaminergic medication has been reported to increment beta-CMC to the level of healthy controls (Salenius et al., 2002).

Motor cortical gamma-band activity $(>30 \mathrm{~Hz})$, on the other hand, peaks after movement onset (Muthukumaraswamy, 2010; Litvak et al., 2012) and is sustained throughout the movement (Crone et al., 1998; Muthukumaraswamy, 2010) and is localized to contralateral motor cortex (Crone et al., 1998). The literature on the effects of DBS and dopaminergic medication on motor cortical gamma activity is sparse, however, STN gamma-band activity has shown concomitant increase with improved motor symptoms after levodopa administration (Brown et al., 2001; Kühn et al., 2006).

In order to elucidate the effects of treatments on motor cortical oscillations and CMC, we measured magnetoencephalography (MEG) and electromyography (EMG) from PD patients in DBS-treated, untreated and dopaminergic medicated states while they performed phasic contractions (hand gripping). We used hand gripping instead of an isometric wrist extension task (which is often used in experiments 
101

\section{Cohort}

Twelve PD patients implanted with STN-DBS at Aarhus University Hospital and 10 healthy controls were recruited for the experiment. All patients had been treated with STN-DBS for at least 6 months. Each participant gave their informed consent and the study was approved by the local ethics committee (The Central Denmark Region Committees on Heath Research Ethics). Exclusion criteria consisted of a MMSE score (Mini-Mental State Examination) below 25 and clinically significant depression as assessed by MDI (Major Depression Inventory). A total of two patients were excluded from the study for reasons extraneous to the exclusion criteria: one patient could not tolerate the DBS OFF conditions while the other had a corrupt EMG channel in one condition and hence did not pass the data quality check. The remaining participant cohorts thus consisted of 10 PD patients (age: $60 \pm 1.7$ (SEM); 5 female) and 10 age-matched healthy controls (age: $58.3 \pm 1.3$ (SEM); 3 female). See Tab. 1 for all relevant demographic and clinical

details on the two cohorts. 


\section{Experimental paradigm}

125 MEG was recorded from participants in supine position with their eyes open. Patients and healthy controls 126 performed self-timed phasic contractions, i.e., grip-relax motions with their right hand, repeatedly over 127 four 30-second sessions with 30 -second breaks in-between as shown in Fig. 1. PD patients had ceased 128 taking dopaminergic medication for $>12$ hours before the experimental sessions. PD patients were 129 recorded in four treatment conditions: DBS-ON, DBS-OFF (0 mins), DBS-OFF (120 mins) and MED-ON 130 conditions. Healthy controls were studied over a similar timescale but without any treatment 131 interventions. Motor symptom assessment (UPDRS-III) was performed before DBS-ON, after DBS-OFF (0 $132 \mathrm{~min}$ ), after DBS OFF (120 $\mathrm{min}$ ) to examine the effects of DBS-washout (Temperli et al., 2003) and before 133 the MED ON condition. The intermediary measurements (DBS OFF (30min) through DBS OFF (90 min)) 134 were available only in some patients and hence were not included in the analysis. Participants received 135 breaks between sessions and were taken in and out of the MEG system whenever possible. The 136 experiment was supervised by a movement disorder specialist. One hour prior to the MED ON condition, 137 PD patients were administered $200 \mathrm{mg}$ of levodopa. In order to ensure that the dopaminergic medication 138 had taken effect before performing the final recording session, UPDRS-III assessments were performed 139 prior to the recording session.

\section{$140 \quad$ MEG acquisition}

141 MEG was recorded with a 306-channel Elekta Neuromag TRIUX MEG system placed in a magnetically 142 shielded room. Apart from the neuromagnetic data, vertical electro-oculogram (vEOG), electromyogram 143 (EMG) of the digitorum communis muscle and electrocardiogram (ECG) were also measured. The data 144 were all recorded with a sampling rate of $1 \mathrm{kHz}$ and a passband of $0.03-330 \mathrm{~Hz}$ (standard settings for the 145 MEG system).

146 Head shape was digitized along with the positions of the anatomical fiducials (nasion and right and left 147 pre-auricular points) and the head position indicator coils (HPIs). The anatomical fiducial positions and 
148 head shape were then used to co-register the participants' MEG data and structural MRIs for source

149 localization. The head position measurements in the MEG system were not possible during the DBS ON

150 condition due to interference from the electrical stimulation with the HPIs. We therefore used the head

151 position measurement from the subsequent recording after switching off DBS and before the patient had

152 been taken out of the MEG system (and thus potentially changed his or her position).

\section{Data preprocessing}

154 The data was first processed using the tSSS algorithm implemented in Maxfilter (Taulu and Simola, 2006)

155 to suppress DBS artefacts and other external magnetic artifacts (subspace correlation limit: 0.9, time 156 window: 10s (Medvedovsky et al., 2009)) and this approach has already been shown to successfully 157 suppress DBS artefacts (Airaksinen et al., 2011, 2012, 2015b, 2015a; Sridharan et al., 2017) and other 158 magnetic interferences (Taulu et al., 2005; Taulu and Simola, 2006; Taulu and Hari, 2009). All data (MEG, 159 EMG, EOG and ECG) were bandpass filtered between 1-100 Hz using a two-pass second order Butterworth 160 filter. An ICA-based artefact rejection routines to suppress ECG- and EOG-related artefacts was 161 implemented using the MNE-Python toolbox (Gramfort et al., 2013). All subsequent analyses of the pre162 processed data were carried out using the Fieldtrip toolbox (Oostenveld et al., 2011) as implemented in 163 MATLAB (The MathWorks Inc., Natick, MA, 2000). Data were epoched into 1-second segments around the 164 peaks of the gripping action. To obtain the peaks, EMG data were Hilbert-transformed and the absolute 165 value was computed to obtain the power envelope after which they were standardized. These 166 standardized envelopes were visually inspected to define thresholds for peaks in each condition which 167 were then used to automatically epoch the data. This epoching procedure rendered and average of $91 \pm 5$ 168 (SEM) epochs in the PD and $97 \pm 2$ in the control cohort (see Tab. 2 for condition-wise details).

\section{Sensor analysis}

170 Power and cross spectral densities (PSD \& CSD) between MEG and EMG data were computed using the 171 multitaper method (Mitra and Pesaran, 1999) with discrete prolate spheroidal sequences (DPSS) as tapers, 
172 as implemented in Fieldtrip. The central frequencies were 1 to $100 \mathrm{~Hz}$ with a bandwidth of $1 \mathrm{~Hz}$. The mean

173 squared coherence was then obtained from the CSD to reflect corticomuscular coherence (CMC). The

174 maxima of the orthogonal gradiometer pairs were used in the case of coherence while the sums of the

175 gradiometer values were used for combining gradiometer pairs for power values. To evaluate the motor

176 output, we computed the mean of the trial-wise root mean square (RMS) which reflects the mean motor

177 output in each condition.

178 The PSDs were normalized by dividing the PSD spectra by the total power from the range of 1-45 $\mathrm{Hz}$ and

179 z-scoring them. We restricted our spectral analysis to below $45 \mathrm{~Hz}$ in order not to confound our analyses

180 with potential DBS artefacts from its first sub-harmonics (i.e. at 65-90 Hz depending on individual

181 stimulation frequency). We defined 15 gradiometer pairs in the left (contralateral) sensorimotor area as

182 the sensors of interest (SOI). We defined two a priori frequency bands of interest: beta (13-30 $\mathrm{Hz})$ and

183 low-gamma (31-45 Hz). We inspected any artefactual peaks in the PSD (due to DBS) and dropped those

184 particular frequency bins from further analysis (see Supplementary material, as well as Airaksinen et al.,

185 (2012), for a similar approach). We obtained summary values for beta-CMC and low-gamma power by

186 searching for maxima in each of the frequency bands in the SOI. The peak frequencies for each subject,

187 condition and measure were passed on to the source localization procedure.

188 Statistical analysis

189 We performed statistics on patient and control cohorts separately with the control cohort mainly serving

190 to account for potential time-related effects (since counter-balancing the conditions was not possible in

191 our experimental design). We used Bayesian inferencing given by Bayes Factors (BF) to test the effects of

192 treatment on UPDRS-III, EMG-RMS, beta-CMC and low-gamma power. BF is an estimate of relative 193 likelihoods of the observed data under the given hypotheses. We used the BayesFactor package (Morey

194 et al., 2015; Sridharan et al., 2017) in R (R Core Team, 2016) to calculate the relevant BFs using the Imbf

195 function along with default settings namely, $r=0.5$ for fixed effects and $r=1$ ('nuisance') for random effects. 
196 In each cohort, we estimated the effect of Condition by modelling a null hypothesis (HO) with only subject-

197 specific offsets as random effects against an unconstrained alternative hypothesis ( $\mathrm{H} 1)$ with Condition as

198 fixed effect and subject-specific offsets as random effects. Hypothesis testing moved progressively

199 towards more constraints and comparing the BFs for each of these equality-constrained hypotheses

200 enabled us to obtain meaningful model-driven statistical inferences on the data. We first framed a more

201 constrained hypothesis $(\mathrm{H} 2)$ to test the three treatment states against each other. We accomplished this

202 by equating DBS OFF (0 min) and DBS OFF (120 min) while allowing DBS ON and MED ON to vary freely.

203 We proceed with testing the specific hypotheses, explained below, only if both $\mathrm{H}_{1} / \mathrm{H}_{0}$ and $\mathrm{H}_{2} / \mathrm{H}_{0}$ showed

204 substantial evidence for the alternative hypothesis.

205 In order to assess whether only one treatment state stood out from the other two, we tested four specific

206 hypotheses with further equality constraints: (H3) equating conditions DBS ON, DBS OFF (0 min) and DBS

207 OFF (120 min) while allowing MED ON to differ; (H4) equating conditions DBS OFF (0 min), DBS OFF (120

$208 \mathrm{~min}$ ) and MED ON while allowing DBS ON to differ; (H5) equating DBS ON and MED ON, as well as DBS

209 OFF (0 $\mathrm{min})$ and DBS OFF (120 min) while allowing the two pairs to differ from each other; and (H6)

210 equating DBS ON and MED ON conditions while allowing DBS OFF (0 min) and DBS OFF (120 min) to vary.

$211 \mathrm{H} 1$ and $\mathrm{H} 2$ in combination with $\mathrm{H} 5$ and $\mathrm{H} 6$ thus implicitly tested for washout effects of the DBS treatment

212 in that the only difference between the two pairs of hypotheses was that both DBS OFF (0 min) and DBS

213 OFF (120 min) were taken to reflect an undifferentiated untreated state in $\mathrm{H} 2$ and $\mathrm{H} 5$ in contrast to $\mathrm{H} 1$

214 and $\mathrm{H} 6$.

\section{Correlations}

216 For both beta-CMC and low-gamma power, we computed their Kendall's tau with both the overall UPDRS-

217 III score and the UPDRS-III bradykinesia (right hand) sub-scores (items: 23 to 25 in the UPDRS scoring

218 manual). We then used the R-function provided along with the recent publication from van Doorn et al.

219 (2016) where the Bayesian inference for correlations in nonparametric data is made on the test statistic 
220 in order to obtain BFs. The resulting BFs for Kendall's tau reflect the relative likelihoods of the observed

221 data under the alternative hypothesis $\left(\mathrm{H}_{1}\right)$ that the modelled slope for the relationship between the two

222 variables (e.g. beta-CMC and UPDRS-III) is non-zero against the null hypothesis $\left(\mathrm{H}_{0}\right)$ that the slope is zero.

223 We computed BFs for five correlations: (i, ii) beta-CMC vs UPDRS-III and UPDRS-III bradykinesia (right

224 hand) sub-scores (items: 23 to 25 in the UPDRS scoring manual) in the MED ON condition; (iii, iv) low-

225 gamma power vs. UPDRS-III and UPDRS-III bradykinesia in the DBS ON condition; and (v) EMG-RMS values

226 vs UPDRS-III scores across all conditions.

\section{Source reconstruction}

228 Based on our observed effects of dopaminergic medication on beta-CMC and of DBS on low-gamma

229 power, we performed source localization only of those two measures in the two treatment conditions,

230 respectively, using beamforming. T1-weighted structural magnetic resonance images (MRI) were acquired

231 for all controls, and we used the pre-operative MRIs for the PD patients. The co-registration of the MEG

232 data and structural MRIs was performed using the fiducials and head shape points recorded during the

233 MEG acquisition.

234 Each subject's structural MRI was segmented and the inner skull volume was non-linearly warped to a

235 canonical MRI included in SPM8 (http://www.fil.ion.ucl.ac.uk/spm/software/spm8/) and canonical source

236 model inverse was warped to obtain the subject-specific beamformer grid (spacing of $5 \mathrm{~mm}$ ) making it

237 comparable across subjects and in correspondence with the specific grid points in the Montreal

238 Neurological Institute (MNI) space (Mattout et al., 2007). The leadfields were computed for each

239 participant and each condition using the single-shell head model based on the individual MRIs of the

240 participants (Nolte, 2003).

241 Source localization was performed using the Dynamic Imaging of Coherent Sources (DICS) method to

242 localize both beta-CMC and low-gamma power (Gross et al., 2001). In the case of beta-CMC, the recorded

243 EMG channel was used as a reference for the source localization procedure. In the case of low-gamma 
244 power, we used the neural activity index (NAI). As already stated, we used the peak frequencies of the

245 beta-CMC and low-gamma power for each subject and condition to focus the source localization. We set

246 the regularization to $5 \%$ for beta-CMC and $0 \%$ for low-gamma power.

247 For visualization purposes, we grand-averaged the beta-CMC and low-gamma power source data in the

248 MED ON and DBS ON conditions, respectively, interpolated and plotted them on a canonical volumetric

249 MRI (Colin 27 brain-1mm resolution) with a threshold of 90\%. We used the AAL atlas (Automated

250 Anatomical Labelling; Tzourio-Mazoyer et al., 2002), as integrated into the Fieldtrip toolbox, as our

251 anatomical reference and in order to obtain MNI coordinates.

252 Results

\section{UPDRS-III}

254 The UPDRS-III scores of the PD cohort are visualized in Fig 2 (summary values in Tab 2). While evaluating 255 the unconstrained hypothesis $\left(\mathrm{H}_{1}\right)$ we obtained a $\mathrm{BF}_{10}=1.42 \mathrm{E} 12$, suggesting that the data were $1.42 \mathrm{E} 12$

256 times more likely to occur under the hypothesis containing Condition as a factor compared to the one

257 without. A BF of this magnitude is considered very strong evidence (Kass and Raftery, 1995) and suggests

258 an effect of Condition on the UPDRS-III scores.

259 The evaluation of the constrained hypotheses $\left(\mathrm{H}_{3-6}\right)$ revealed highest BF for $\mathrm{H}_{6}$ where DBS ON and MED

260 ON conditions were equated while allowing DBS OFF (0 $\mathrm{min})$ and DBS OFF (120 $\mathrm{min})$ conditions to vary.

261 Compared to the unconstrained hypothesis $\left(\mathrm{H}_{1}\right)$, the data were 8.4 times more likely $\left(\mathrm{BF}_{61}=8.43\right)$ to occur

262 under this constrained hypothesis, and 7,375 times more likely $\left(\mathrm{BF}_{65}=7,375\right)$ to occur under this hypothesis

263 compared to the hypothesis $\left(\mathrm{H}_{5}\right)$ with DBS ON and MED ON equated and DBS OFF (0 min) and DBS OFF

264 (120 min) equated. These are considered positive evidence and very strong evidence (Kass and Raftery,

265 1995), respectively, and suggest that the two treatments (dopaminergic medication and DBS) lead to

266 similar ameliorating effects on the motor symptoms, while the washout effect of the DBS treatment on 
267 the motor symptoms are reflected in the increasing UPDRS-III scores between DBS OFF (0 min) and DBS

268 OFF (120 min) (See Tab.2 for Summary values).

\section{EMG-RMS}

270 The mean of the EMG-RMS of both cohorts are plotted in Fig. 2 (summary values Tab. 2). Evaluation of

271 the unconstrained hypothesis $\left(\mathrm{H}_{1}\right)$ showed that the EMG-RMS was 177 times $\left(\mathrm{BF}_{10}=177.38\right)$ more likely to

272 arise from this hypothesis with Condition as a factor compared to the hypothesis without $\left(\mathrm{H}_{0}\right)$. This is

273 considered very strong evidence (Kass and Raftery, 1995) and suggests an effect of Condition on EMG-

274 RMS.

275 In evaluation of the specific hypotheses $\mathrm{H}_{3-6}$, we obtained the highest BF for $\mathrm{H}_{5}$ where DBS ON and MED

276 ON as well as DBS OFF (0 min) and DBS OFF (120 min) were equated. The data were 3.3 times $\left(\mathrm{BF}_{51}=3.33\right)$

277 more likely to occur under $\mathrm{H}_{5}$ compared to the unconstrained hypothesis $\left(\mathrm{H}_{1}\right)$. This is considered positive

278 evidence (Kass and Raftery, 1995) and suggests that the PD patients' EMG-RMS, and hence their motor

279 output, were similar in the two sets of conditions, i.e. in DBS ON and MED ON, as well as in the untreated

280 state with DBS OFF (0 min) and DBS OFF (120 min). The corresponding BFs for Controls showed evidence

281 for the data under the null hypothesis, i.e. for no effects of Condition (see Tab. 3).

\section{Sensor analysis}

$283 C M C$

284 The group means and the grand averaged topographies of beta-CMC are shown in Fig. 3a. The grand

285 averages of the beta-CMC showed relatively focal effects in a few sensors over the contralateral

286 sensorimotor area (See Fig. 3b). The grand-averaged coherence spectra based on the sensor that captured

287 the maximal beta-CMC is shown in Fig. 3c. The grand-averaged spectra and the spectra at the individual

288 level showed no artefactual peaks due to DBS. Evaluating the effects of treatments on beta-CMC revealed

289 that the beta-CMC data were 3.5 times $\left(\mathrm{BF}_{10}=3.45\right)$ more likely to arise from the hypothesis containing 
Condition as a factor and specified as the three treatment states $\left(\mathrm{H}_{1}\right)$ compared to the null hypothesis

without Condition as a factor $\left(\mathrm{H}_{0}\right)$. This is considered positive evidence (Kass and Raftery, 1995) suggesting an effect of treatment on beta-CMC.

On evaluating the specific hypotheses $\left(\mathrm{H}_{3-6}\right)$, we found the highest BF for $\mathrm{H}_{3}$ where DBS ON, DBS OFF $(0$

times $\left(\mathrm{BF}_{30}=14.23\right)$ more likely to arise from this hypothesis compared to the null hypothesis $\left(\mathrm{H}_{0}\right)$ and 2 conditions and thus that dopaminergic medication affects beta-CMC. The corresponding BFs for the

\section{Power}

Fig. 4a shows the group means and grand-averaged topographies of low-gamma power. In the DBS ON condition in the PD patients, the low-gamma power showed a focus in activity in sensors over the contralateral sensorimotor area (Fig. 4b), quite similar in distribution to the beta-CMC. The grandaveraged power spectra based on the sensor that captured the maximal low-gamma power is shown in

Fig. 4c. Individual power spectra showed subject-specific artefactual frequency bins within the frequency range of interest, which were dropped from the analysis (see Methods). Evaluating the effect of treatment under the hypothesis with Condition as a factor and with the DBS OFF (0 min) and DBS OFF (120 min) equated $\left(\mathrm{H}_{2}\right)$ compared to the null hypothesis. This is considered very strong evidence (Kass and Raftery,

311 1995) suggesting an effect of treatment on low-gamma power. Evaluation of the specific hypotheses $\left(\mathrm{H}_{3-}\right.$

312 6) revealed that the low-gamma power data were 6728 times $\left(\mathrm{BF}_{40}=6727.95\right)$ more likely to occur under 
314 null hypothesis $\left(\mathrm{H}_{0}\right)$. This is considered very strong evidence (Kass and Raftery, 1995) and suggests that

315 the low-gamma power is different in the DBS-treated condition compared to the other three conditions

316 and thus an effect of DBS on movement-related low-gamma power. The corresponding BFs for the

317 Controls showed evidence for the null hypothesis and thus suggest no effects of Condition (or Time, see

318 Tab. 3).

319 In order to rule out any potential effects in the DBS ON condition due to confounds from sub-harmonics

320 and interference from the electrical stimulation (at $130 \mathrm{~Hz}$ ), we conducted a parallel sham-analysis for

321 low-gamma power with 15 gradiometer pairs over the right (ipsilateral) hemisphere and with a set of 8

322 gradiometer pairs over the occipital region. Low-gamma summary values obtained from the right and

323 occipital SOls did not show any modulation by the treatments, thereby confirming that the increase in

324 low-gamma power in DBS ON over the sensorimotor area is very unlikely to be an artefact of the electrical

325 stimulation (see Supplementary material). We also correlated the DBS voltage levels for the patients' left

326 and right hemispheres with their observed low-gamma power over the contralateral sensorimotor area

327 as a further test for potential confounds from the electrical stimulation (See Correlations).

328 Correlations

329 UPDRS-bradykinesia (right-hand) sub-scores correlated with the beta-CMC in the MED ON condition

$330 \quad\left(\mathrm{BF}_{10}=3.83 ; \tau=-0.57\right)$, suggesting that the observed data were 3.8 times more likely to occur under the

331 alternative $\left(\mathrm{H}_{1}\right)$ than under the null hypothesis $\left(\mathrm{H}_{0}\right)$. The overall UPDRS-III scores did not show any

332 substantial evidence for the data under the alternative or the null hypothesis $\left(B F_{10}=1.86 ; \tau=-0.47\right)$. While

333 the former BF (UPDRS-bradykinesia) is considered positive evidence, the latter BF (UPDRS-III) denotes

334 evidence barely worth a mention (Kass and Raftery, 1995). The low-gamma power was not correlated with

335 the overall UPDRS-III scores $\left(\mathrm{BF}_{10}=0.63 ; \tau=-0.26\right)$ or the UPDRS-bradykinesia (right-hand) sub-scores

$336\left(\mathrm{BF}_{10}=0.59 ; \tau=0.24\right)$. The $\mathrm{BFs}$ suggest that the observed relationships between the low-gamma power and

337 the UPDRS-III and UPDRS-bradykinesia scores were 1.6 and 1.7 times more likely to occur under the null 
hypothesis $\left(\mathrm{H}_{0}\right)$ with zero slope, respectively. Neither BFs are considered more than evidence barely worth a mention (Kass and Raftery, 1995).

340 The UPDRS-III scores correlated with the motor output as measured by EMG-RMS $\left(\mathrm{BF}_{10}=132.75\right.$;

$341 \tau=-0.41)$. The BF suggests that the observed data were 133 times more likely to occur under the

342 alternative hypothesis $\left(\mathrm{H}_{1}\right)$ with non-zero slope than under the null hypothesis $\left(\mathrm{H}_{0}\right)$ with zero slope. This

343 is considered very strong evidence (Kass and Raftery, 1995).

344 For the potentially confounding correlations between DBS voltage levels and low-gamma power, we did

345 not find any substantial evidence for or against the data under the null or the alternative hypotheses.

346 When modelling the correlations between DBS voltage and low-gamma power in the DBS ON condition,

347 the BFs suggested weak evidence for the data under the null hypothesis $\left(H_{0}\right)\left(\right.$ left: $B F_{10}=0.39, \tau=0.03$; right:

$\left.348 \mathrm{BF}_{10}=0.48, \tau=-0.17\right)$. The low-gamma power results thus do not seem to be confounded by the electrical

349 stimulation, albeit the correlation analyses only provide weak evidence for this conclusion (see

350 Supplementary material).

\section{Source reconstruction}

352 Fig. 5 shows the grand-averaged source localization maps of the beta-CMC and low-gamma power in the

353 PD cohort in the MED ON and DBS ON conditions, respectively. The maxima of the cortical source were

354 localized to the precentral gyrus (motor cortex) in the left hemisphere, contralateral to the active

355 muscular movement for both metrics ( $\mathrm{MNI}$ coordinates of the peaks of the grand averaged source

356 estimates: beta-CMC in MED ON condition: $x=-32 ; y=0 ; z=44$; low-gamma power in DBS ON condition:

$357 \quad x=-32 ; y=-18 ; z=58)$.

358 Discussion

359 We have shown in this study, differentiated effects of DBS and dopaminergic medication on movement

360 related oscillations in the motor cortex and the corticomuscular interaction while both treatments 
361 showing similar ameliorating effects on the motor symptoms in PD patients. Dopaminergic medication

362 augments beta-CMC (13-30 Hz) in PD patients (localized to contralateral precentral gyrus) while DBS

363 increases low-gamma $(31-45 \mathrm{~Hz})$ spectral power (also localized to contralateral precentral gyrus).

\section{Mechanism of dopaminergic medication on beta-CMC and motor symptoms}

365 The beta-CMC in the medicated state was correlated with improvements in the UPDRS-III scores and the UPDRS-bradykinesia (right hand) sub-scores, indicating a possible link between the general improvement of motor symptoms through dopaminergic medication and an increase in beta-CMC. Studies with healthy subjects have shown that better performance with fewer errors are accompanied by increased beta-CMC (Kristeva et al., 2007; Witte et al., 2007; van Wijk et al., 2012) indicating that higher CMC may lead to better compliance and performance.

371 While the literature on DBS effects on beta-CMC have shown mixed effects with some indicating a 372 significant increase in beta-CMC due to DBS (Park et al., 2009) and others reporting no group effect of DBS 373 on beta-CMC, pointing to high inter-individual differences in the effect of DBS (Airaksinen et al., 2015b).

374 Dopaminergic medication seems to increase beta-CMC to the levels of healthy controls (Salenius et al., 375 2002), though Hirschmann et al. (2013) report no significant effects of dopaminergic medication on beta376 CMC. Our results are thus in line with the increased beta-CMC during MED ON reported by Salenius et al. 377 (Salenius et al., 2002), though in comparison with healthy controls we report an overshoot in the betaCMC. Such differences between the two studies may arise from differences in the nature of the tasks 379 applied. While Salenius et al. (Salenius et al., 2002) used an isometric contraction task, we used a phasic hand gripping task, as it has been shown that $\mathrm{CMC}$ varies depending on the nature of the task (Kilner et medication (Hirschmann et al., 2013), it is important to note that the experiment in said report were performed with subcutaneous apomorphine medication, and although it has a very short half-life and was 
finding. So we compliment the results previously reported by eradicating the potentially confounding incorporating a DBS ON condition.

In rat models, dopamine has been shown to affect local circuits and the cortical interneuron activity which medication in PD patients.

\section{Mechanism of DBS on low-gamma power and motor symptoms}

DBS, unlike dopaminergic medication, seems to amplify movement-related low-gamma (31-45 Hz) power

403 facilitates movement (Brown et al., 2001; Kühn et al., 2006; Cheyne and Ferrari, 2013) and that 404 dopaminergic medication increases gamma activity in the STN in combination with improvement of motor 405 symptoms suggesting a potential role for gamma activity in the basal ganglia-cortical loop (Brown et al.,

406 2001; Kühn et al., 2006). Increased activity in the low-gamma band is indicative of the activity of the 407 underlying neuronal population (Manning et al., 2009; Miller et al., 2009) and the increased low-gamma 
band activity during DBS ON might indicate a higher level of activation of the motor cortex during movement (Muthukumaraswamy, 2010).

We speculate that our reported effect of STN-DBS on movement-related low-gamma power arise through

411 antidromic activation of a "hyperdirect" pathway between M1 and STN (Nambu et al., 2002; Gradinaru et

412 al., 2009; Nambu and Tachibana, 2014). Substantial projections from deep cortical layers to the ipsilateral

413 dorsolateral area of STN have been found in monkey (Nambu et al., 2000) and in humans (Fernandez-

414 Miranda et al., 2012; Petersen et al., 2017), supporting the existence of such a hyperdirect pathway. The

415 central role of the antidromic activation of the hyperdirect pathway between M1 and STN in the

416 therapeutic effect of DBS has been made plausible through optogenetic studies (Gradinaru et al., 2009)

417 and computational studies (Kang and Lowery, 2014). It has also been shown that cortical oscillatory

418 activity is functionally coupled to STN oscillations through phase-amplitude coupling, which is in turn

419 affected by DBS (Whitmer et al., 2012; de Hemptinne et al., 2013; Shimamoto et al., 2013). Hence, DBS

420 potentially modulates the cortical low-gamma oscillations through its effects on the basal ganglia-cortical

421 functional network via the "hyperdirect pathway".

422 However, we note that the present study was performed with a modest cohort of 10 PD patients and 10

423 healthy controls, and the evidence for our observed treatment effects on movement related cortical

424 oscillations are moderate in nature and thus necessitate studies with larger cohorts to ensure the

425 potential reproducibility of the results.

426 In conclusion, we have shown differentiated effects of DBS and dopaminergic medication on cortical

427 activity during phasic hand contractions. While both treatments affect motor symptoms similarly, each of

428 the treatments may employ different functional modulation strategies through differentiated anatomical

429 pathways in their improvement of the motor output. 


\section{Acknowledgements}

431 We thank our PD patients and healthy controls who participated in the study. We would like to thank

432 Sarang Dalal for his advice on the source analysis, Lau Møller Andersen and Mads Jensen for their advice

433 on the MEG pre-processing, and Jonas Kristoffer Lindeløv for his advice on the statistical analyses. This

434 study is based on work that has been funded by the Danish Research Council for Independent Research,

435 the Danish Parkinson Association, Central Denmark Research Foundation, Aarhus University and Aarhus

436 University Hospital.

437 Conflict of Interest

$K \emptyset$ : Consultancy for Metdtronic Inc.; Honororia from Medtronic Inc., UCB, Fertin Pharma and AbbVie outside the submitted work. SB: non-financial support from Elekta and EGI; personal fees from UCB Pharma outside the submitted work.

\section{$441 \quad$ Figure captions}

442 Fig.1. Experimental paradigm: MEG measurements are represented by the vertical bars and each measurement session reflects $\sim 2$ minutes of phasic contractions. UPDRS-III was performed at four time

444 points marked by the grey arrows. The background colors represent the three treatment states, namely 445 DBS-treated (orange), untreated (green) and dopaminergic medication-treated (blue) states. Controls did 446 not receive any treatment. Experiment cohorts consisted of 10 PD patients and 10 healthy controls each.

447 See Methods section for more details.

448 Fig.2. Clinical motor state and motor output: (a) Group averages of the UPDRS-III (motor part) scores of 449 the PD cohort are shown at the top (broken maroon line) together with EMG-RMS (motor output) of the 450 PD (solid red) and control cohorts (solid green) at the bottom. Higher values of UPDRS-III scores indicate 451 worsening motor symptoms while higher EMG-RMS values indicate better motor output. While UPDRS-III 
453 a washout effect of DBS (DBS OFF (0 min) compared to DBS OFF (120 min)). See Results section for more

454 details. (b) The Hilbert envelope of the epoched EMG trials are plotted along with the trial-averaged

455 envelope (thick line) for each condition from a representative patient (PD\#2). See Methods section for

456 more details.

Fig.3. Beta-CMC - trends and topographies: (a) Changes in beta-CMC during phasic contractions in the PD (red line) and control (green line) cohorts. The * sign denotes the condition that was found to differ substantially from the others in the PD cohort according to our statistical analyses. Errorbars reflect \pm SEM. (b) Topographic plots of the group-averaged condition-wise beta-CMC for the PD (top row) and control

461 (bottom row) cohorts. Source localization confirmed that the beta-CMC during MED ON was localized to 462 the contralateral sensorimotor area (see Fig. 5). See Methods section for more details. (c) The grand averaged coherence spectra based on the sensor which captured the maximal coherence in each subject

464 and condition are shown.

Fig.4. Low-gamma power - trends and topographies: (a) Changes in low-gamma power during phasic contractions for the PD (red line) and control (green line) cohorts. The * sign denotes the condition that

467 was found to be different substantially from the others in the PD cohort according to our statistical 468 analyses. Errorbars reflect \pm SEM. (b) Topographic plots of the group-averaged condition-wise low-gamma 469 power for the PD (top row) and control (bottom row) cohorts. Source localization confirmed that the low470 gamma power during DBS ON was localized to the contralateral sensorimotor area (see Fig. 5). (c) The 471 grand averaged power spectra based on the sensor which captured the maximal low-gamma power in 472 each subject and condition are shown.

473 Fig.5. Source estimates of beta-CMC and low-gamma power: Group-averaged source estimates of the 474 beta-CMC (top row) in the MED ON condition and of the low-gamma power (bottom row) in the DBS ON 475 condition. Low-gamma power was localized to contralateral M1. 


\section{Table captions}

477 Tab. 1. Details of the PD and control cohorts: DBS: deep brain stimulation; MED: L-Dopa medication;

478 UPDRS-III: Unified Parkinson's Disease Rating Scale (motor part). SR: slow release; LEDD: levodopa

479 equivalent daily dosage; LEDD formula: $100 \mathrm{mg}$ of levodopa $=130 \mathrm{mg}$ of controlled-release levodopa $=70$

$480 \mathrm{mg}$ of levodopa + COMT-inhibitor $=1 \mathrm{mg}$ of pramipexole $=5 \mathrm{mg}$ of ropinirole (Mamikonyan et al., 2008).

481 " denotes that the UPDRS-III scoring in those conditions was performed before the experiment session.

482 Tab. 2. Summary values: Means and SEMs of the differences between measures in each condition and

483 the DBS OFF (120 min) condition, thus used as the reference condition. The SEMs of the group averages

484 in the reference condition are shown in parentheses in that column. The different conditions favored by

485 the statistical analyses are highlighted in bold.

Tab. 3. Bayes Factors: Bayes Factors for the corresponding alternative hypotheses against the null. Bayes

487 Factors suggesting the strongest evidence for or against the null hypothesis for a given measure are highlighted in bold. ' 1 ' denotes DBS ON; '2' denotes DBS OFF (0 min); ' 3 ' denotes DBS OFF (120 min); and

'4' denotes MED ON. From Kass and Raftery (Kass and Raftery, 1995): BF = 1 to 3 is considered evidence

"barely worth a mention"; $\mathrm{BF}=3$ to 20 is considered "positive evidence"; $\mathrm{BF}=20$ to 150 is considered

"strong evidence"; BF > 150 is considered "very strong evidence".

\section{Supplementary material}

493 Supp. Fig. S1: DBS artefact rejection: Power spectra of a sample PD patient (PD\#4) during DBS ON and

494 MED ON conditions from three SOls (left and right hemispheres and the occipital area). Bins showing sharp

495 peaks occurring in all three SOls (here at $34 \mathrm{~Hz}$ ) were considered artefactual and dropped from further 496 analysis (see Methods). 
Supp. Fig. S2: Correlation between beta-CMC and clinical motor state: Scatter plots of the beta-CMC and

UPDRS-III (left panel) and UPDRS-III-hypokinesia sub-scores from the right hand (right panel) in the MED

ON condition. The corresponding BFs and the estimated correlation coefficients are in the titles of the respective plots. While there is positive evidence for a negative correlation between beta-CMC and the UPDRS-III-bradykinesia sub-scores, the evidence in the case of UPDRS-III scores is barely worth a mention. See Correlations section for more details.

Supp. Fig. S3: Influence of DBS artefacts on low-gamma power: (a) Scatter plots of the low-gamma power and the estimated correlation coefficients are in the titles of the respective plots. While both BFs denote evidence barely worth a mention for zero-slope, indicating no substantial evidence for the data under the null hypothesis, there is even less substantial evidence for the data under the alternative hypothesis. (b) Low-gamma power in the occipital region across the four conditions. The plot shows no substantial differences in low-gamma power in the occipital region between treatments. This suggests that the increased low-gamma power in the DBS ON condition is a localized effect over the sensorimotor area and that it is not due to corruption from sub-harmonics of the DBS artefacts. hypotheses against the null for the sham SOls, namely, the right hemisphere and the occipital area.

514 Evaluation of the hypothesis $\left(\mathrm{H}_{1}\right)$ revealed that the low-gamma power data was more likely to occur under 515 the null hypothesis compared to the alternative hypothesis with Conditions as factor $\left(\mathrm{H}_{1}\right)$. This is 516 considered positive evidence (Kass and Raftery, 1995) and suggests that the low-gamma power was not 517 substantially different between conditions and thus no noticeable effect of DBS treatment on movement518 related low-gamma power over the right hemisphere and over the occipital area. This confirms that the 519 increase in low-gamma power in DBS ON over the left sensorimotor area is very unlikely to be an artefact of the electrical stimulation. 


\section{References}

522 Airaksinen K, Butorina A, Pekkonen E, Nurminen J, Taulu S, Ahonen A, Schnitzler A, Mäkelä JP (2012)

523 Somatomotor mu rhythm amplitude correlates with rigidity during deep brain stimulation in

$524 \quad$ Parkinsonian patients. Clin Neurophysiol 123:2010-2017.

525 Airaksinen K, Lehti T, Nurminen J, Luoma J, Helle L, Taulu S, Pekkonen E, Mäkelä JP (2015a) Corticomuscular coherence parallels coherence of postural tremor and MEG during static muscle contraction. Neurosci Lett 602:22-26.

Airaksinen K, Mäkelä JP, Nurminen J, Luoma J, Taulu S, Ahonen A, Pekkonen E (2015b) Cortico-muscular coherence in advanced Parkinson's disease with deep brain stimulation. Clin Neurophysiol

Airaksinen K, Mäkelä JP, Taulu S, Ahonen A, Nurminen J, Schnitzler A, Pekkonen E (2011) Effects of DBS on auditory and somatosensory processing in Parkinson's disease. Hum Brain Mapp 32:1091-1099.

Baker SN, Chiu M, Fetz EE, Fetz Afferent EE (2006) Afferent Encoding of Central Oscillations in the Monkey Arm. J Neurophysiol 95:3904-3910.

Baker SN, Olivier E, Lemon RN (1997) Coherent oscillations in monkey motor cortex and hand muscle EMG show task-dependent modulation. J Physiol 501:225-241.

Brown P, Oliviero a, Mazzone P, Insola a, Tonali P, Di Lazzaro V (2001) Dopamine dependency of oscillations between subthalamic nucleus and pallidum in Parkinson's disease. J Neurosci 21:10331038. 
542 Crone NE, Miglioretti DL, Gordon B, Lesser RP (1998) Functional mapping of human sensorimotor cortex

543 with electrocorticographic spectral analysis. II. Event-related synchronization in the gamma band.

$544 \quad$ Brain 121:2301-2315.

545 de Hemptinne C, Ryapolova-Webb ES, Air EL, Garcia P a, Miller KJ, Ojemann JG, Ostrem JL, Galifianakis

546 NB, Starr P a (2013) Exaggerated phase-amplitude coupling in the primary motor cortex in

$547 \quad$ Parkinson disease. Proc Natl Acad Sci U S A 110:4780-4785.

548 Deuschl G et al. (2006) A randomized trial of deep-brain stimulation for Parkinson's disease. N Engl J

$549 \quad$ Med 355:896-908.

550 Fernandez-Miranda JC, Pathak S, Engh J, Jarbo K, Verstynen T, Yeh FC, Wang Y, Mintz A, Boada F,

551 Schneider W, Friedlander R (2012) High-definition fiber tractography of the human brain:

552 Neuroanatomical validation and neurosurgical applications. Neurosurgery 71:430-453.

553 Gao W-J, Goldman-Rakic PS (2003) Selective modulation of excitatory and inhibitory microcircuits by 554 dopamine. Proc Natl Acad Sci U S A 100:2836-2841.

555 Gao W-J, Wang Y, Goldman-Rakic PS (2003) Dopamine modulation of perisomatic and peridendritic

556 inhibition in prefrontal cortex. J Neurosci 23:1622-1630.

557 Gradinaru V, Mogri M, Thompson KR, Henderson JM, Deisseroth K (2009) Optical deconstruction of 558 parkinsonian neural circuitry. Science 324:354-359.

559 Gramfort A, Luessi M, Larson E, Engemann DA, Strohmeier D, Brodbeck C, Goj R, Jas M, Brooks T, 560 Parkkonen L, Hämäläinen M (2013) MEG and EEG data analysis with MNE-Python. Front Neurosci $7: 267$.

562 Gross J, Kujala J, Hamalainen M, Timmermann L, Schnitzler A, Salmelin R (2001) Dynamic imaging of 563 coherent sources: Studying neural interactions in the human brain. Proc Natl Acad Sci U S A 
Gwin JT, Ferris DP (2012) Beta- and gamma-range human lower limb corticomuscular coherence. Front Hum Neurosci 6:1-6.

Hammond C, Bergman H, Brown P (2007) Pathological synchronization in Parkinson's disease: networks, models and treatments. Trends Neurosci 30:357-364.

Hirschmann J, Özkurt TE, Butz M, Homburger M, Elben S, Hartmann CJ, Vesper J, Wojtecki L, Schnitzler A (2013) Differential modulation of STN-cortical and cortico-muscular coherence by movement and levodopa in Parkinson's disease. Neuroimage 68:203-213.

572 Hughes AJ, Daniel SE, Kilford L, Lees AJ, Daniel SE (1992) Accuracy of clinical diagnosis of idiopathic Parkinson's disease: a clinico-pathological study of 100 cases. Neurosurgery, and Psychiatry

Johnsen EL, Mogensen PH, Sunde NA, Østergaard K (2009) Improved asymmetry of gait in Parkinson's disease with DBS: Gait and postural instability in Parkinson's disease treated with bilateral deep

Kang G, Lowery MM (2014) Effects of antidromic and orthodromic activation of STN afferent axons brain stimulation in the subthalamic nucleus. Mov Disord 24:590-597.

Kass RE, Raftery AE (1995) Bayes Factors. J Am Stat Assoc 90:773-795.

Kilner JM, Baker SN, Salenius S, Hari R, Lemon RN (2000) Human cortical muscle coherence is directly related to specific motor parameters. J Neurosci 20:8838-8845.

Kristeva R, Patino L, Omlor W (2007) Beta-range cortical motor spectral power and corticomuscular coherence as a mechanism for effective corticospinal interaction during steady-state motor output. Neuroimage 36:785-792. 
586 Kühn A a., Kupsch A, Schneider GH, Brown P (2006) Reduction in subthalamic 8-35 Hz oscillatory activity

587 correlates with clinical improvement in Parkinson's disease. Eur J Neurosci 23:1956-1960.

Lang AE, Lozano AM (1998) Parkinson's Disease. N Engl J Med 339:1044-1053.

Limousin P, Krack P, Pollak P, Benazzouz A, Ardouin C, Hoffmann D, Benabid A-L (1998) Electrical

\section{0}

591 Stimulation of the Subthalamic Nucleus in Advanced Parkinson's Disease. N Engl J Med 339:1105-

Litvak V, Eusebio A, Jha A, Oostenveld R, Barnes G, Foltynie T, Limousin P, Zrinzo L, Hariz MI, Friston K, Brown P (2012) Movement-Related Changes in Local and Long-Range Synchronization in Parkinson's Disease Revealed by Simultaneous Magnetoencephalography and Intracranial

Mamikonyan E, Siderowf AD, Duda JE, Potenza MN, Horn S, Stern MB, Weintraub D (2008) Long-term follow-up of impulse control disorders in Parkinson's disease. Mov Disord 23:75-80.

Manning JR, Jacobs J, Fried I, Kahana MJ (2009) Broadband shifts in local field potential power spectra are correlated with single-neuron spiking in humans. J Neurosci 29:13613-13620.

Marsden JF, Werhahn KJ, Ashby P, Rothwell J, Noachtar S, Brown P (2000) Organization of cortical activities related to movement in humans. J Neurosci 20:2307-2314.

Mattout J, Henson RN, Friston KJ (2007) Canonical source reconstruction for MEG. Comput Intell Neurosci 2007.

McIntyre CC, Hahn PJ (2010) Network perspectives on the mechanisms of deep brain stimulation. Neurobiol Dis 38:329-337. 
Medvedovsky M, Taulu S, Bikmullina R, Ahonen A, Paetau R (2009) Fine tuning the correlation limit of spatio-temporal signal space separation for magnetoencephalography. J Neurosci Methods $177: 203-211$.

Miller KJ, Zanos S, Fetz EE, den Nijs M, Ojemann JG (2009) Decoupling the cortical power spectrum reveals real-time representation of individual finger movements in humans. J Neurosci 29:31323137.

Mitra PP, Pesaran B (1999) Analysis of dynamic brain imaging data. Biophys J 76:691-708.

Morey RD, Rouder JN, Jamil T (2015) BayesFactor: Computation of Bayes Factors for Common Design. R package.

Muthukumaraswamy SD (2010) Functional properties of human primary motor cortex gamma oscillations. J Neurophysiol 104:2873-2885.

Nambu A, Tachibana Y (2014) Mechanism of parkinsonian neuronal oscillations in the primate basal ganglia: some considerations based on our recent work. Front Syst Neurosci 8:74.

Nambu A, Tokuno H, Hamada I, Kita H, Imanishi M, Akazawa T, Ikeuchi Y, Hasegawa N (2000) Excitatory cortical inputs to pallidal neurons via the subthalamic nucleus in the monkey. J Neurophysiol 84:289-300.

Nambu A, Tokuno H, Takada M (2002) Functional significance of the cortico- subthalamo- pallidal ‘ hyperdirect ' pathway. Neuro Res 43:111-117.

Nolte G (2003) The magnetic lead field theorem in the quasi-static approximation and its use for magnetoencephalography forward calculation in realistic volume conductors. Phys Med Biol 48:3637-3652.

Oostenveld R, Fries P, Maris E, Schoffelen J-M (2011) FieldTrip: Open Source Software for Advanced 
631

632

Analysis of MEG, EEG, and Invasive Electrophysiological Data. Comput Intell Neurosci 2011:1-9.

$\varnothing$ stergaard K, Aa Sunde N (2006) Evolution of Parkinson's disease during 4 years of bilateral deep brain stimulation of the subthalamic nucleus. Mov Disord 21:624-631.

Park H, Kim JS, Paek SH, Jeon BS, Lee JY, Chung CK (2009) Cortico-muscular coherence increases with tremor improvement after deep brain stimulation in Parkinson's disease. Neuroreport 20:14441449.

Petersen M V., Lund TE, Sunde N, Frandsen J, Rosendal F, Juul N, Østergaard K (2017) Probabilistic versus deterministic tractography for delineation of the cortico-subthalamic hyperdirect pathway in patients with Parkinson disease selected for deep brain stimulation. J Neurosurg 126:16571668.

Pollok B, Krause V, Martsch W, Wach C, Schnitzler A, Südmeyer M (2012) Motor-cortical oscillations in early stages of Parkinson's disease. J Physiol 590:3203-3212.

R Core Team (2016) R: A Language and Environment for Statistical Computing.

Riddle CN, Baker SN (2005) Manipulation of peripheral neural feedback loops alters human corticomuscular coherence. J Physiol 566:625-639.

Salenius S, Avikainen S, Kaakkola S, Hari R, Brown P (2002) Defective cortical drive to muscle in Parkinson's disease and its improvement with levodopa. Brain 125:491-500.

Salenius S, Portin K, Kajola M, Salmelin R, Hari R (1997) Cortical control of human motoneuron firing during isometric contraction. J Neurophysiol 77:3401-3405.

Schnitzler A, Gross J (2005) Normal and pathological oscillatory communication in the brain. Nat Rev Neurosci 6:285-296.

Shimamoto S a, Ryapolova-Webb ES, Ostrem JL, Galifianakis NB, Miller KJ, Starr P a (2013) Subthalamic 
nucleus neurons are synchronized to primary motor cortex local field potentials in Parkinson's disease. J Neurosci 33:7220-7233.

Sridharan KS, Højlund A, Johnsen EL, Sunde NA, Johansen LG, Beniczky S, Østergaard K (2017) Parkinson's disease. Clin Neurophysiol 128:1327-1336.

Surmeier DJ, Ding J, Day M, Wang Z, Shen W (2007) D1 and D2 dopamine-receptor modulation of striatal glutamatergic signaling in striatal medium spiny neurons. Trends Neurosci 30:228-235.

Taulu S, Hari R (2009) Removal of magnetoencephalographic artifacts with temporal signal-space separation: demonstration with single-trial auditory-evoked responses. Hum Brain Mapp 30:1524-

Taulu S, Simola J (2006) Spatiotemporal signal space separation method for rejecting nearby interference in MEG measurements. Phys Med Biol 51:1759-1768. Process 53:3359-3372.

Temperli P, Ghika J, Villemure J-G, Burkhard PR, Bogousslavsky J, Vingerhoets FJG (2003) How do parkinsonian signs return after discontinuation of subthalamic DBS? Neurology 60:78-81. (2002) Automated anatomical labeling of activations in SPM using a macroscopic anatomical parcellation of the MNI MRI single-subject brain. Neuroimage 15:273-289. 
bioRxiv preprint doi: https://doi.org/10.1101/657882; this version posted June 3, 2019. The copyright holder for this preprint (which was not certified by peer review) is the author/funder, who has granted bioRxiv a license to display the preprint in perpetuity. It is made available under

675 Van Wijk BCM, Litvak V, Friston KJ, Daffertshofer a. (2013) Nonlinear coupling between occipital and

676 motor cortex during motor imagery: A dynamic causal modeling study. Neuroimage 71:104-113.

677 Whitmer D, de Solages C, Hill B, Yu H, Henderson JM, Bronte-Stewart H (2012) High frequency deep

678 brain stimulation attenuates subthalamic and cortical rhythms in Parkinson's disease. Front Hum

$679 \quad$ Neurosci 6:1-18.

680 Witham CL, Riddle CN, Baker MR, Baker SN (2011) Contributions of descending and ascending pathways

681 to corticomuscular coherence in humans. J Physiol 589:3789-3800.

682 Witte M, Patino L, Andrykiewicz A, Hepp-Reymond MC, Kristeva R (2007) Modulation of human

683 corticomuscular beta-range coherence with low-level static forces. Eur J Neurosci 26:3564-3570. 


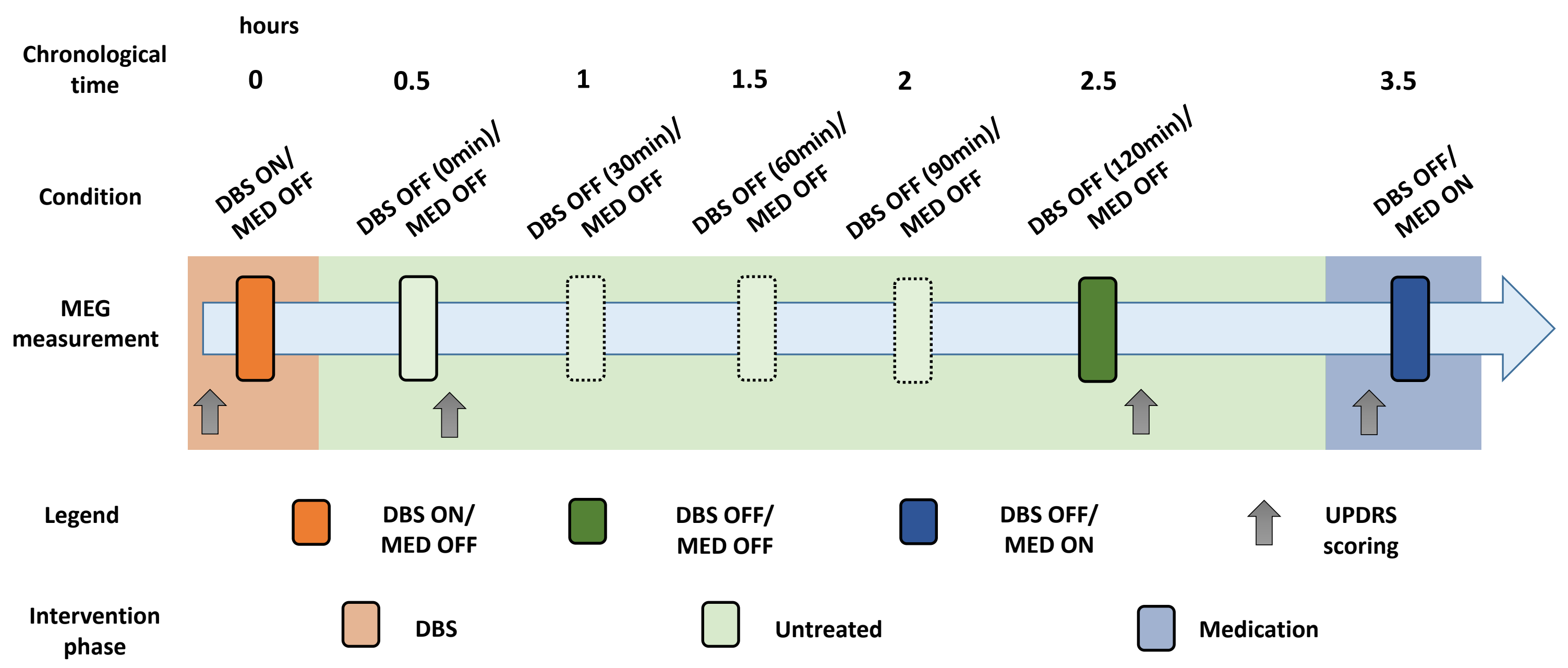


bioRxiv preprint doi: https://doi.org/10.1101/657882; this version posted June 3, 2019. The copyright holder for this preprint (which was not

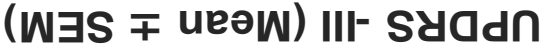
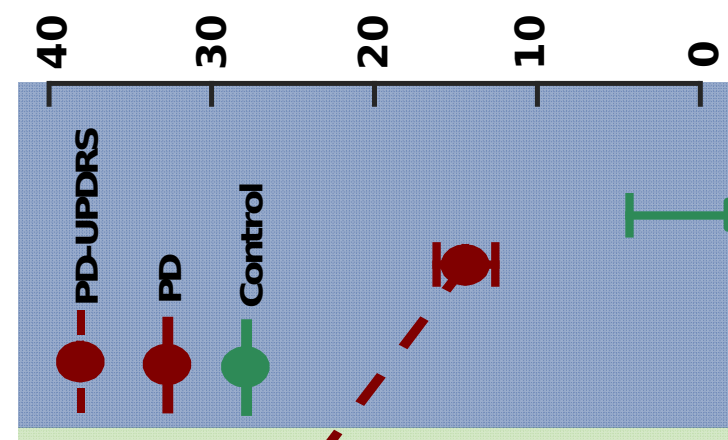

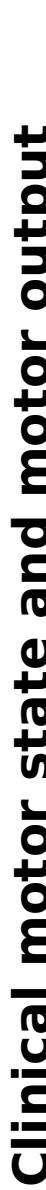

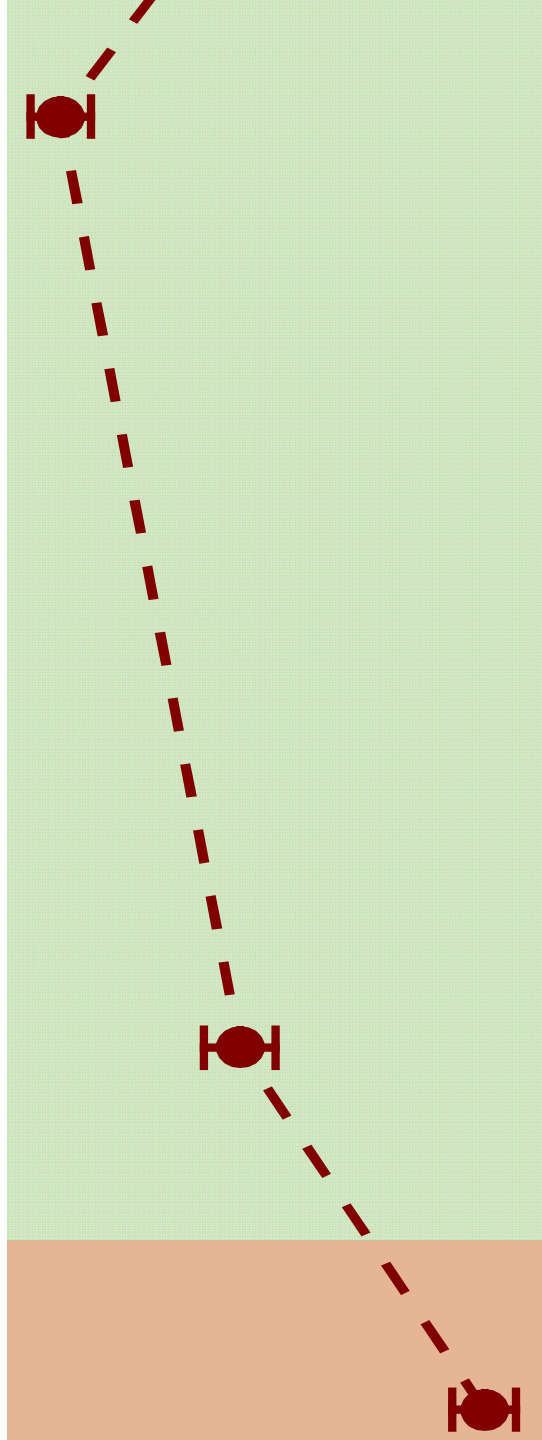

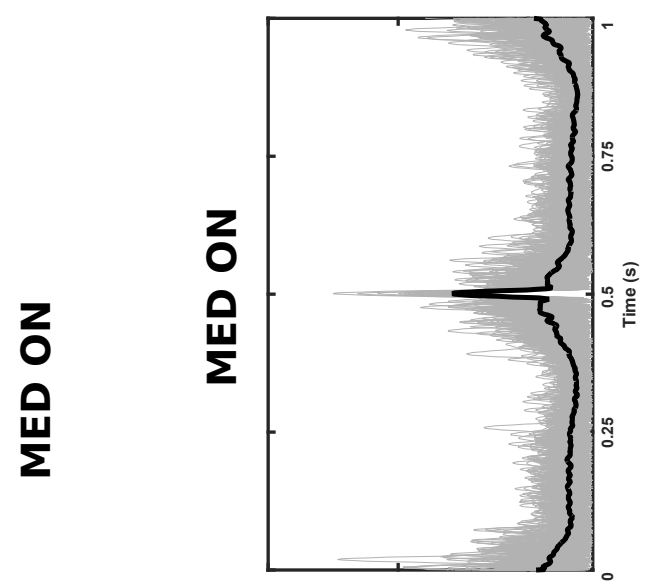
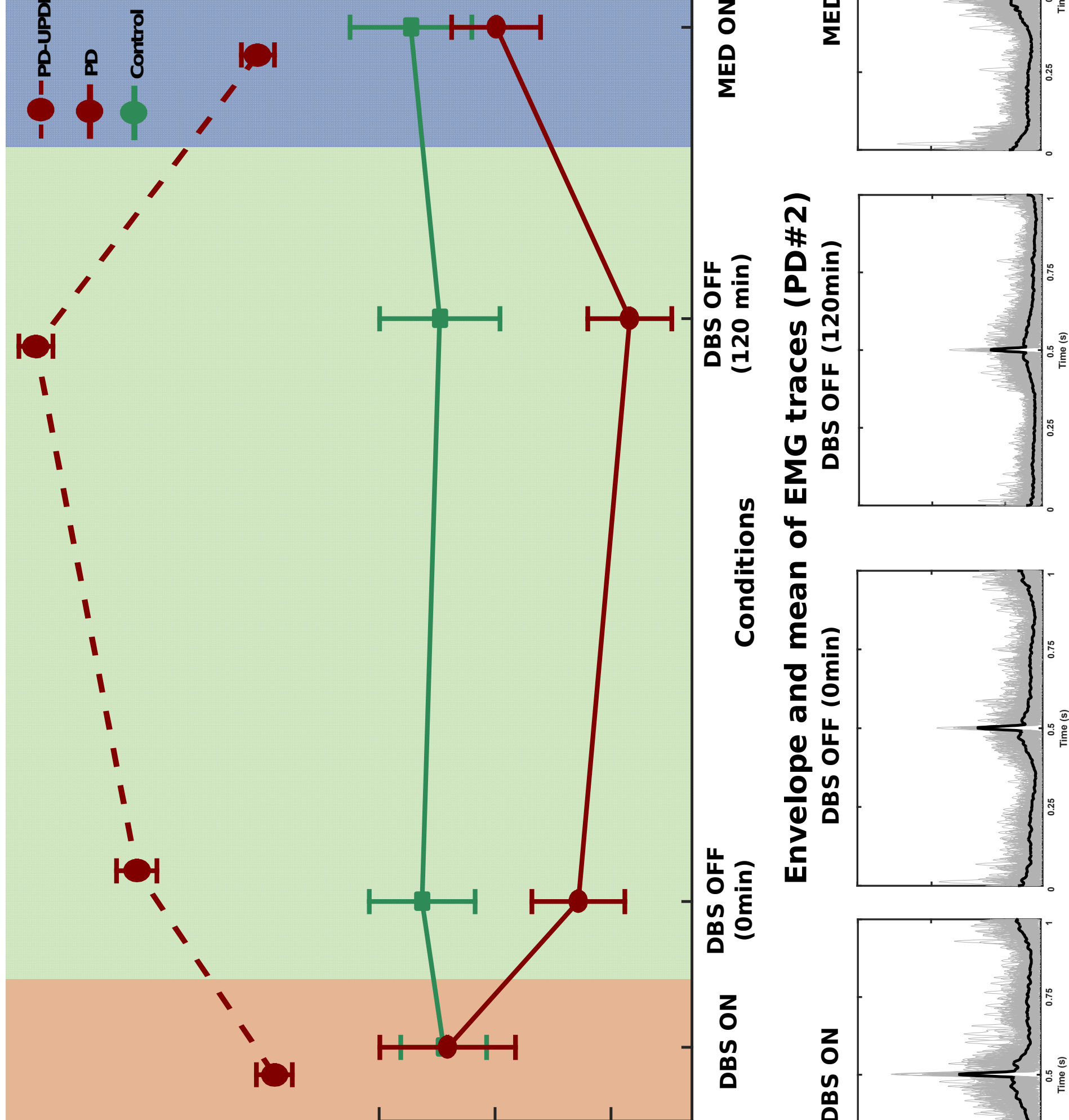

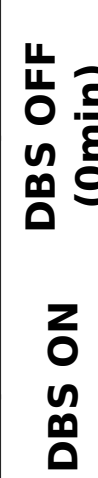

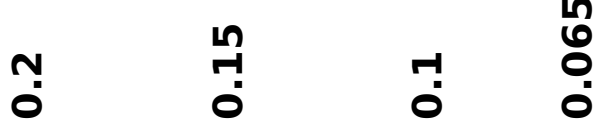

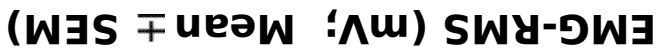

응

$\frac{4}{0}$
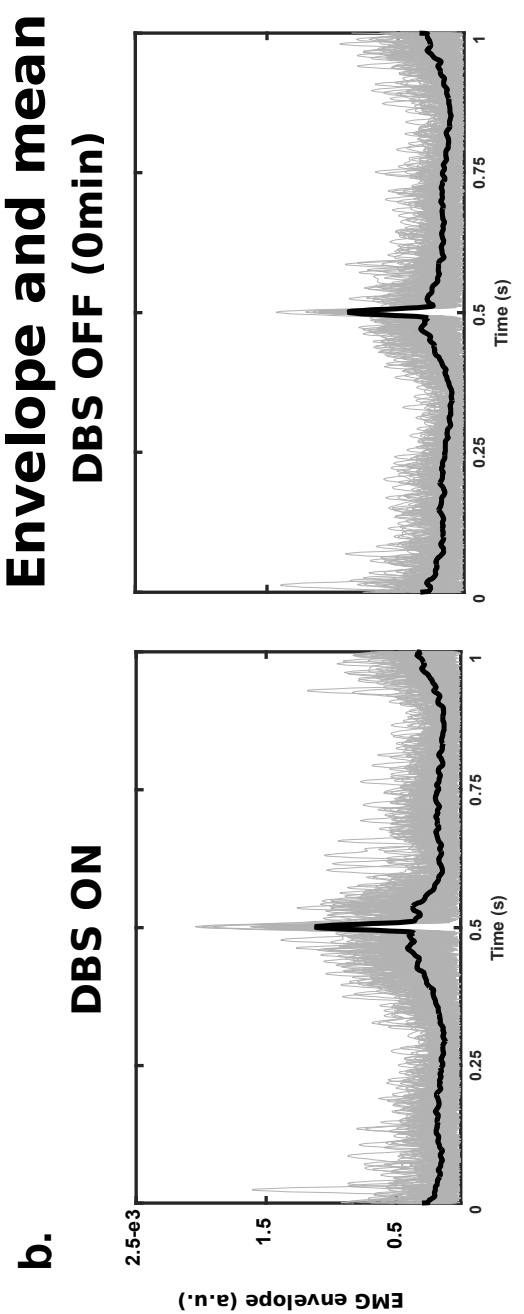


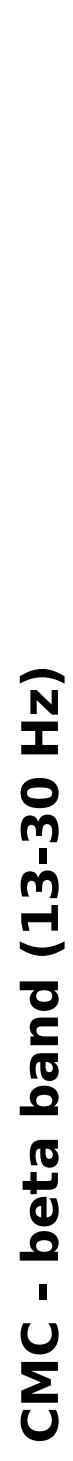

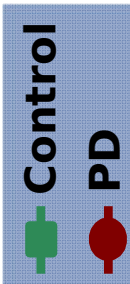
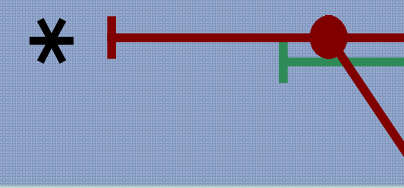

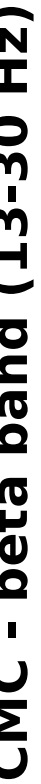

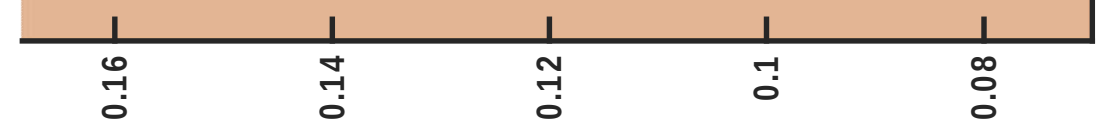

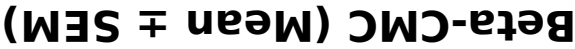

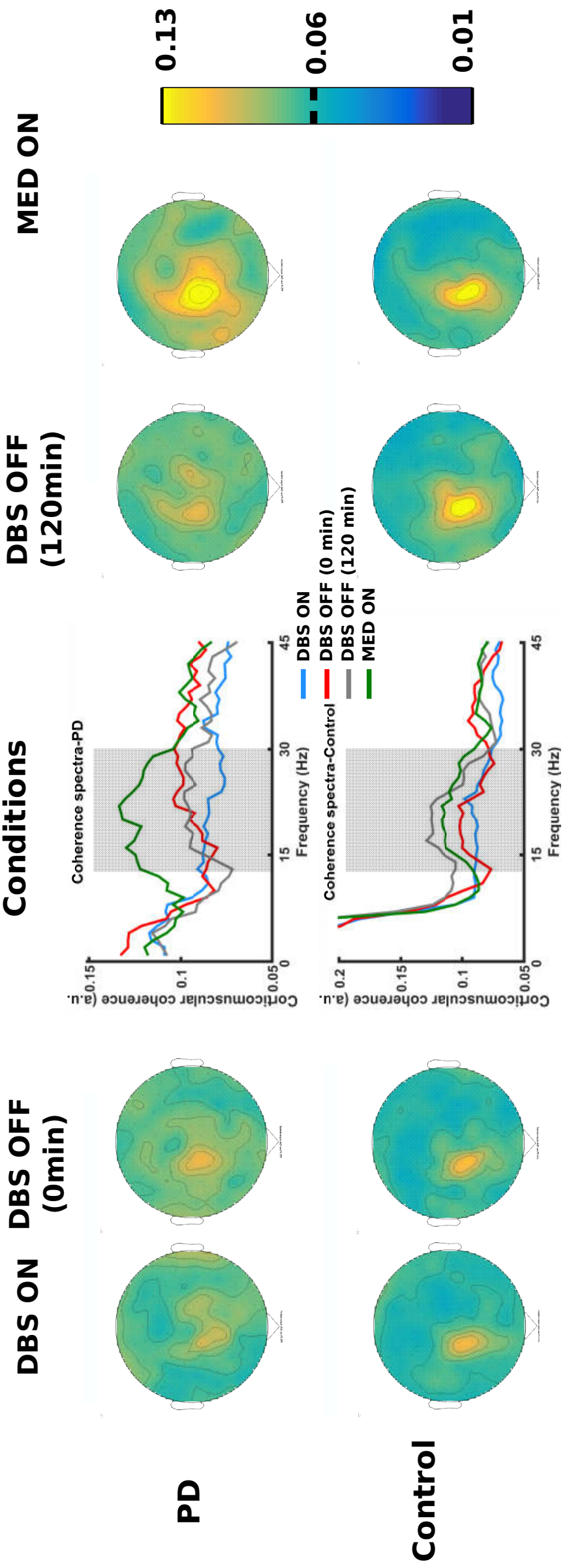



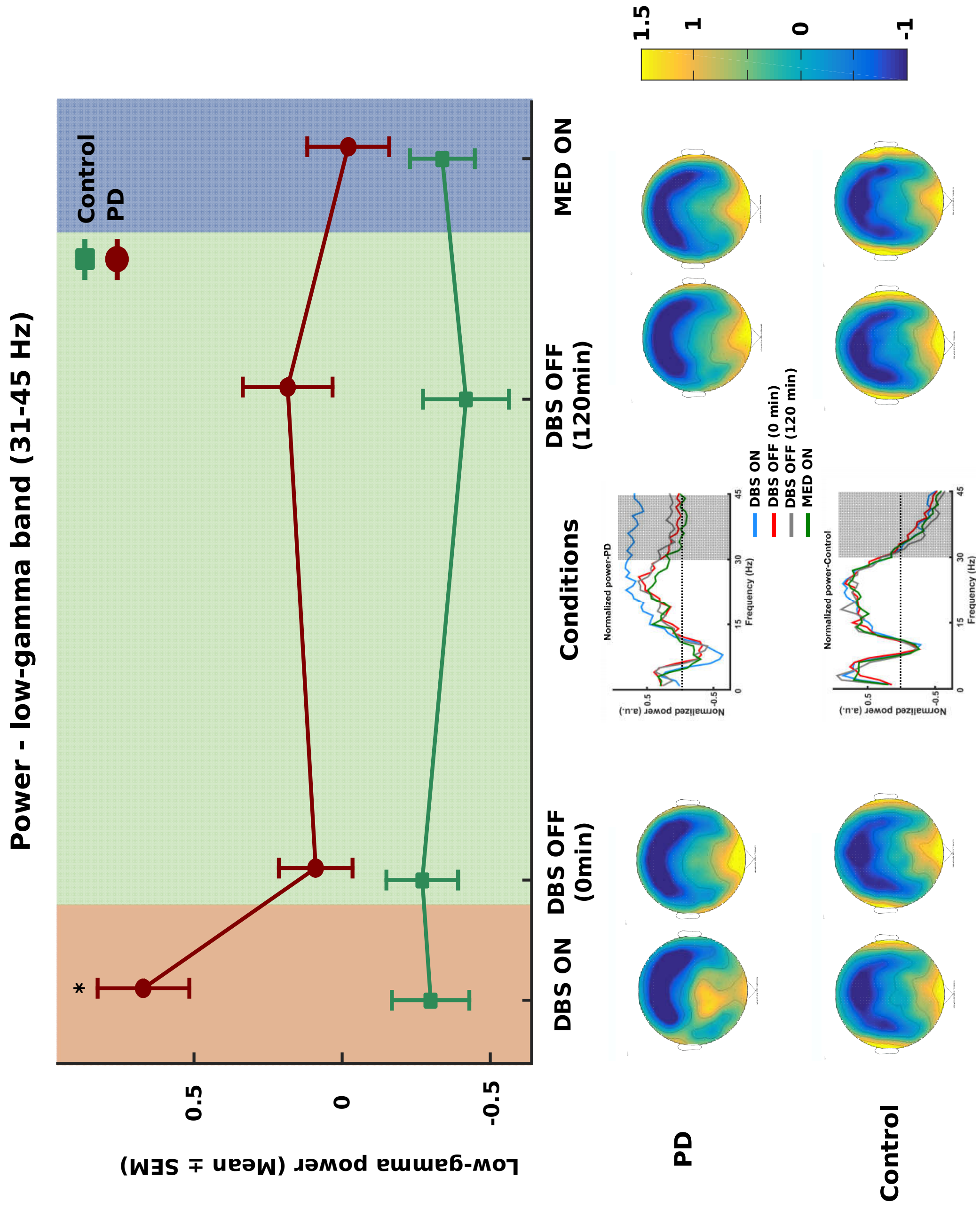
Fig.5. Source localization of beta-CMC and low-gamma power

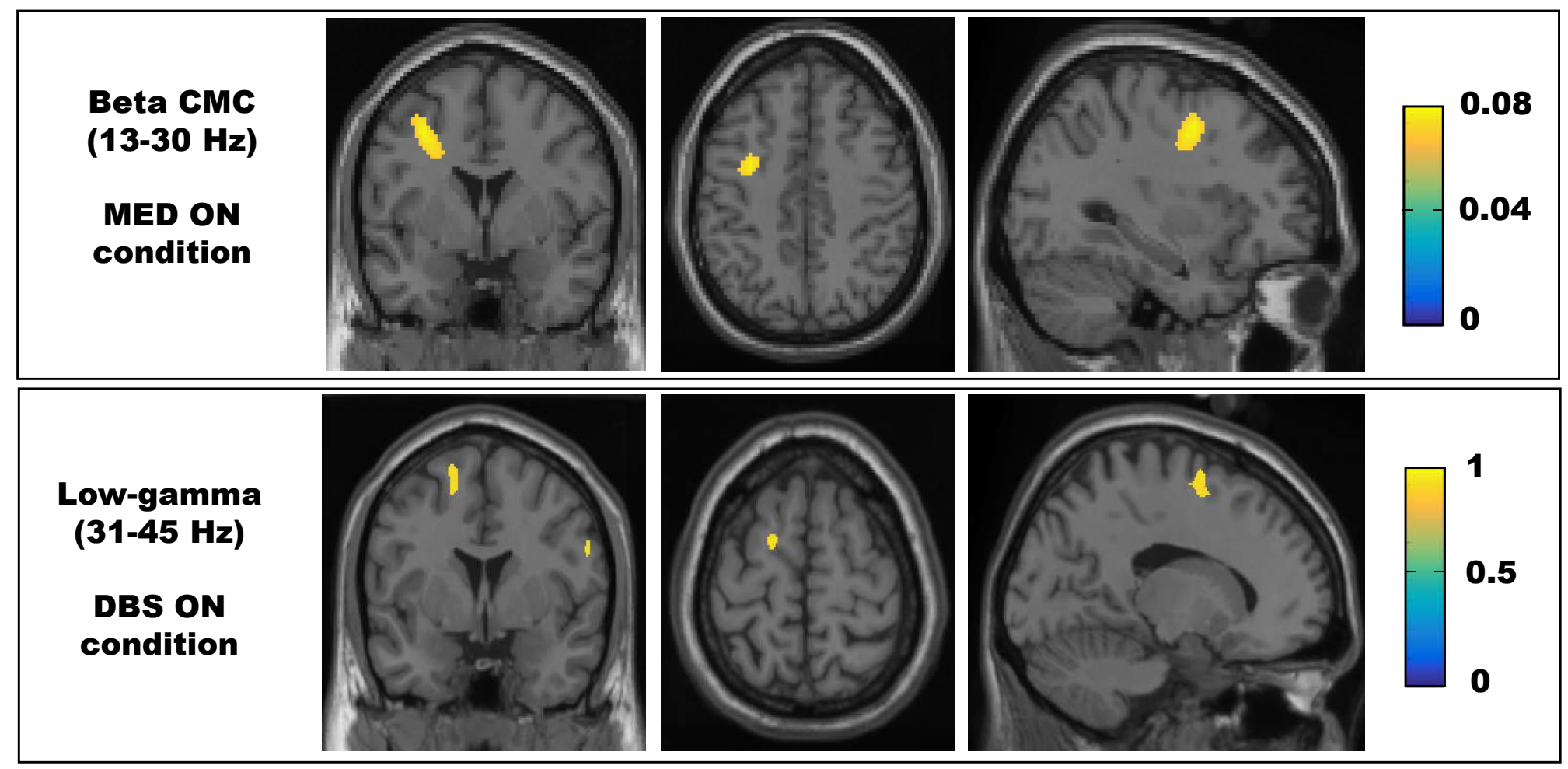




\begin{tabular}{|c|c|c|c|c|c|c|c|c|c|c|c|c|c|c|c|}
\hline \multirow[b]{2}{*}{ ID } & \multirow[b]{2}{*}{ Age } & \multirow[b]{2}{*}{ Gender } & \multirow[b]{2}{*}{ MDI } & \multirow[b]{2}{*}{ MMSE } & \multicolumn{2}{|c|}{ Dopa-agonist } & \multicolumn{2}{|c|}{ L-Dopa } & \multirow[b]{2}{*}{$\begin{array}{l}\text { LEDD } \\
\text { (mg) }\end{array}$} & \multicolumn{2}{|c|}{ Stimulation settings } & \multicolumn{4}{|c|}{ UPDRS } \\
\hline & & & & & Drug & $\begin{array}{c}\text { Dosage } \\
\text { (mg) }\end{array}$ & Drug & $\begin{array}{l}\text { Dosage } \\
\text { (mg) }\end{array}$ & & Left STN & Right STN & $\begin{array}{l}\text { DBS } \\
\text { ON }\end{array}$ & $\begin{array}{c}\text { DBS } \\
\text { OFF } \\
(\text { Omin })^{\#}\end{array}$ & $\begin{array}{l}\text { DBS OFF } \\
(120 \mathrm{~min})^{\#}\end{array}$ & $\begin{array}{l}\text { MED } \\
\text { ON }\end{array}$ \\
\hline PD1 & 53 & $\mathrm{~F}$ & $\mathrm{~N}$ & 29 & Pramipexole & 0.7 & Stalevo & 400 & 470 & 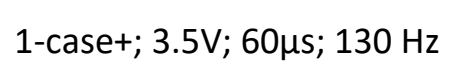 & 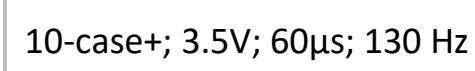 & 13 & 31 & 40 & 7 \\
\hline PD2 & 61 & $\mathrm{~F}$ & $\mathrm{~N}$ & 29 & Ropinirole (SR) & 10 & Madopar & 375 & 575 & 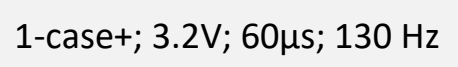 & 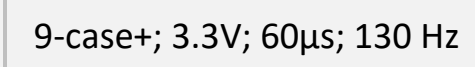 & 12 & 16 & 29 & 14 \\
\hline PD3 & 62 & $\mathrm{M}$ & $\mathrm{N}$ & 29 & Ropinirole (SR) & 8 & Madopar & 400 & 560 & 2-case+; 3.6V; 60us; $130 \mathrm{~Hz}$ & 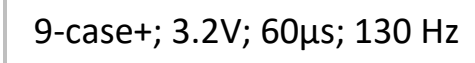 & 12 & 23 & 31 & 11 \\
\hline PD4 & 50 & $\mathrm{~F}$ & $\mathrm{~N}$ & 29 & - & - & $\begin{array}{l}\text { Sinemet/ } \\
\text { Madopar }\end{array}$ & 700 & 700 & 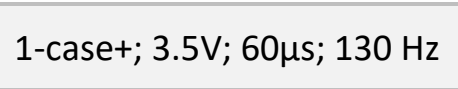 & 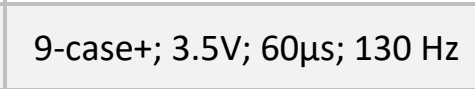 & 12 & 30 & 39 & 13 \\
\hline PD5 & 66 & M & $\mathrm{N}$ & 29 & Pramipexole & 1.05 & Sinemet & 200 & 305 & 1-case+; $2.3 \mathrm{~V} ; 60 \mu \mathrm{s} ; 130 \mathrm{~Hz}$ & 9-case+; 3.1V; 60us; $130 \mathrm{~Hz}$ & 11 & 21 & 40 & 15 \\
\hline PD6 & 59 & M & $\mathrm{N}$ & 30 & - & - & Sinemet & 300 & 300 & 1-case+; $2.4 \mathrm{~V} ; 60 \mu \mathrm{s} ; 130 \mathrm{~Hz}$ & 9 -case+; $2.7 \mathrm{~V} ; 60 \mu \mathrm{s} ; 130 \mathrm{~Hz}$ & 5 & 26 & 40 & 16 \\
\hline PD7 & 59 & M & $\mathrm{N}$ & 30 & - & - & Sinemet & 600 & 600 & 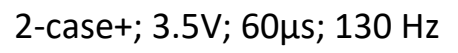 & 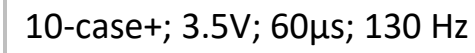 & 14 & 30 & 38 & 21 \\
\hline PD8 & 60 & $\mathrm{~F}$ & $\mathrm{~N}$ & 29 & Ropinirole (SR) & 6 & Sinemet & 250 & 370 & 1-case+; 3.5V; 60us; $130 \mathrm{~Hz}$ & 9-case+; 3.5V; 60us; $130 \mathrm{~Hz}$ & 6 & 24 & 35 & 5 \\
\hline PD9 & 68 & $\mathrm{M}$ & $\mathrm{N}$ & 29 & - & - & Sinemet & 700 & 700 & 1-case+; $2.5 \mathrm{~V} ; 60 \mu \mathrm{s} ; 130 \mathrm{~Hz}$ & 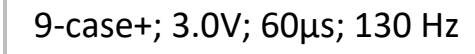 & 28 & 37 & 46 & 20 \\
\hline PD10 & 62 & $\mathrm{~F}$ & $\mathrm{~N}$ & 30 & - & - & $\begin{array}{l}\text { Sinemet/ } \\
\text { Madopar }\end{array}$ & 400 & 400 & 1-case+; 3.5V; $60 \mu \mathrm{s} ; 130 \mathrm{~Hz}$ & 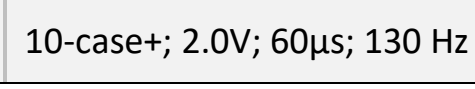 & 13 & 38 & 48 & 22 \\
\hline C1 & 62 & $\mathrm{~F}$ & $\mathrm{~N}$ & 30 & & & & & & & & & & & \\
\hline C2 & 54 & $\mathrm{M}$ & $\mathrm{N}$ & 30 & & & & & & & & & & & \\
\hline C3 & 58 & $\mathrm{~F}$ & $\mathrm{~N}$ & 30 & & & & & & & & & & & \\
\hline C4 & 64 & M & $\mathrm{N}$ & 30 & & & & & & & & & & & \\
\hline C5 & 51 & $\mathrm{M}$ & $\mathrm{N}$ & 29 & & & & & & & & & & & \\
\hline C6 & 56 & $\mathrm{~F}$ & $\mathrm{~N}$ & 30 & & & & & & & & & & & \\
\hline C7 & 55 & $\mathrm{M}$ & $\mathrm{N}$ & 30 & & & & & & & & & & & \\
\hline C8 & 59 & $\mathrm{M}$ & $\mathrm{N}$ & 30 & & & & & & & & & & & \\
\hline C9 & 63 & $\mathrm{M}$ & $\mathrm{N}$ & 28 & & & & & & & & & & & \\
\hline C10 & 61 & $\mathrm{M}$ & $\mathrm{N}$ & 30 & & & & & & & & & & & \\
\hline
\end{tabular}




\begin{tabular}{|c|c|c|c|c|c|c|c|c|c|}
\hline \multirow{2}{*}{\multicolumn{2}{|c|}{ Measures }} & \multicolumn{4}{|c|}{ PD } & \multicolumn{4}{|c|}{ Control } \\
\hline & & \multirow{3}{*}{$\begin{array}{c}\Delta \text { DBS ON } \\
-0.012 \\
0.008\end{array}$} & \multirow{2}{*}{$\begin{array}{c}\begin{array}{c}\Delta \text { DBS OFF } \\
\text { (0min) }\end{array} \\
0.001\end{array}$} & \multirow{2}{*}{$\begin{array}{c}\text { DBS OFF } \\
(120 \mathrm{~min}) \\
0.094( \pm 0.01)\end{array}$} & \multirow{3}{*}{$\begin{array}{c}\Delta \text { MED ON } \\
0.030 \\
0.013\end{array}$} & \multirow{3}{*}{$\begin{array}{c}\Delta \text { DBS ON } \\
-0.024 \\
0.015\end{array}$} & \multirow{3}{*}{$\begin{array}{c}\begin{array}{c}\Delta \text { DBS OFF } \\
\text { (0min) }\end{array} \\
-0.020 \\
0.017\end{array}$} & \multirow{2}{*}{$\begin{array}{c}\begin{array}{c}\text { DBS OFF } \\
(120 \mathrm{~min})\end{array} \\
0.110( \pm 0.025)\end{array}$} & \multirow{3}{*}{$\begin{array}{c}\Delta \text { MED ON } \\
-0.003 \\
0.015\end{array}$} \\
\hline & Mean & & & & & & & & \\
\hline & SEM & & 0.007 & & & & & & \\
\hline \multirow{2}{*}{ Low-gamma Power } & Mean & 0.487 & -0.095 & $0.184( \pm 0.15)$ & -0.205 & 0.119 & 0.147 & $-0.418( \pm 0.14)$ & 0.079 \\
\hline & SEM & 0.129 & 0.118 & & 0.129 & 0.061 & 0.095 & & 0.106 \\
\hline \multirow{2}{*}{ UPDRS - III } & Mean & -26.0 & -11.0 & $38.6( \pm 1.9)$ & -24.2 & & & & \\
\hline & SEM & 2.1 & 1.1 & & 5.5 & & & & \\
\hline \multirow{2}{*}{$\begin{array}{c}\text { EMG-RMS } \\
(\mathrm{mv})\end{array}$} & Mean & 0.79 & 0.22 & $0.919( \pm 0.574)$ & 0.58 & -0.02 & 0.08 & $1.73( \pm 0.259)$ & 0.12 \\
\hline & SEM & 0.20 & 0.35 & & 0.09 & 0.21 & 0.18 & & 0.06 \\
\hline \multirow{2}{*}{ Number of trials } & Mean & 2 & -11 & $93( \pm 9)$ & 1 & 1 & 1 & $95( \pm 4)$ & 2 \\
\hline & SEM & 13 & 13 & & 8 & 4 & 2 & & 4 \\
\hline
\end{tabular}




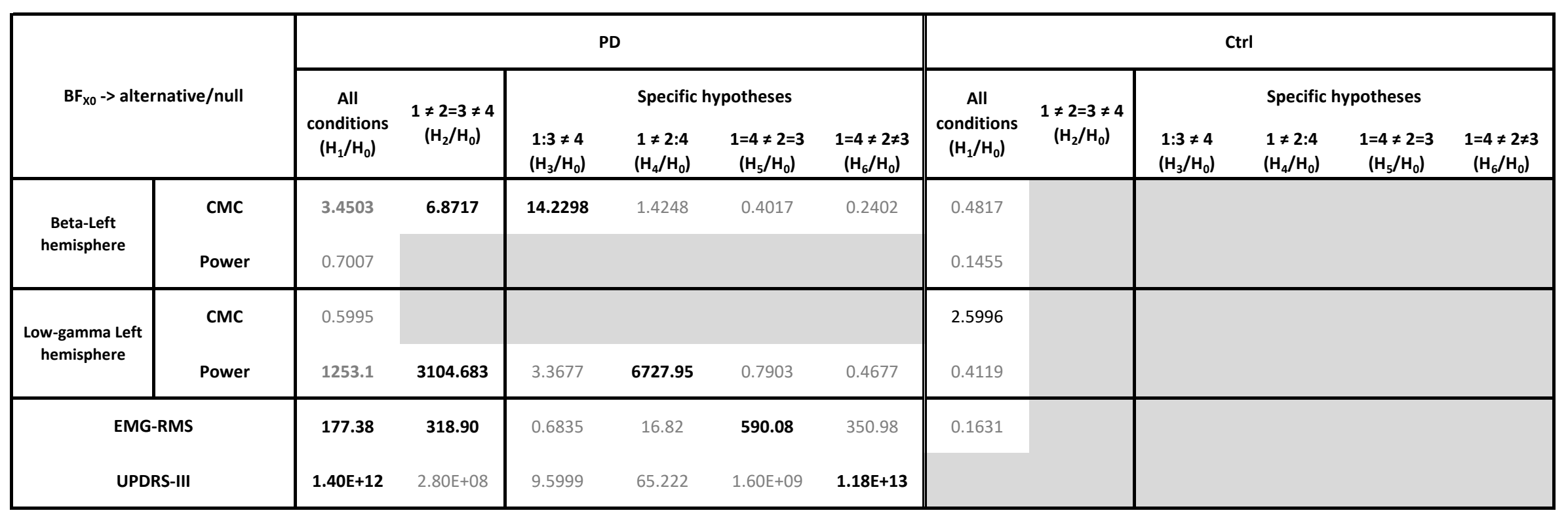

